\title{
Phenotypic and molecular characterization of the causal agent of chafer beetle mortality in the wheat fields of the Kurdistan province, Iran
}

\author{
Keivan Karimi ${ }^{1 *}$, Mahdi Arzanlou${ }^{1}$, Asadollah Babai Ahari ${ }^{1}$, Mostafa Mansour Ghazi²
}

\author{
${ }^{1}$ Plant Protection Department, Agricultural Faculty, University of Tabriz, P.O. Box 5166614766, Tabriz, Iran \\ ${ }^{2}$ Plant Protection Department, Agricultural and Natural Resources Research Center of Kurdistan, P.O. Box 714, \\ Sanandaj, Iran
}

Received: September 9, 2014

Accepted: June 19, 2015

\begin{abstract}
We report the first case of chafer beetle [Anisoplia austriaca (Herbst 1783)] mortality caused by Actinomucor elegans var. elegans in wheat fields of the Kurdistan province, Iran. For three years, dead larvae of Anisoplia austriaca were collected from wheat fields of the Kurdistan province. Similar isolates of a fast-growing fungus were recovered from all samples. The fungal isolates were identified as A. elegans var. elegans based on morphological and cultural characteristics. The identity of the species was further confirmed using sequence data of the ITS (Internal Transcribed Spacer) region of ribosomal DNA. Koch's postulates were fulfilled by the inoculation of the larvae of A. austriaca and Galleria mellonella (Linnaeus, 1758) (as the model insect) using the spore suspension of A. elegans var. elegans. The viability of sporangiospores was evaluated using a spore dilution technique on germination medium. The results on the pathogenicity (100\% mortality in A. austriaca larvae) and viability tests (germination: $95.45 \%)$ demonstrated that A. elegans var. elegans can be considered as a potential biocontrol agent against the chafer beetle. Field experiments are still required to evaluate the capacity of A. elegans as a biological control agent.
\end{abstract}

Key words: Actinomucor elegans var. elegans, biocontrol, chafer beetle, mortality

\section{Introduction}

Of all the cereals, wheat (Triticum aestivum L.) is the most valuable and strategic crop. Wheat serves as a staple food commodity in major parts of the world, especially in the Middle East region including Iran. Wheat production is affected by many biotic and abiotic factors. Diseases (e.g. rusts, fusarium head blight) and pests (e.g. sun pest) can cause a significant reduction of the yield.

Wheat chafer beetle [Anisoplia austriaca (Herbst, 1783) Scarabidae] is one of the important pests of wheat. As an adult, this beetle feeds on plant pollen or immature plant seeds, and as larvae feed on wheat roots (Jameson et al. 2007). These feeding practices result in yield losses. In general, scarab beetles are likely to increase in significance because of, for one, the decreasing availability of conventional chemical insecticides. Unfortunately, there are very few microorganisms commercially available that can effectively and economically replace the broad spectrum chemical insecticides used against these pests (Klein and Kaya 1995). Based on these facts, and due to the large beetle population, and wheat damage caused by the chafer beetle on wheat in the Kurdistan province, the aim of the present study was to survey the natural enemies of the chafer beetle in wheat fields in the Kurdistan province, Iran. Dead larvae of chafer beetle were frequently observed in wheat fields. The causal agent was characterized using morphological and molecular data. The pathogenic potential of this agent was further evaluated on healthy larvae of A. austriaca and Galleria mellonella (Linnaeus, 1758) in laboratory conditions.

\section{Materials and Methods}

\section{Monitoring and microorganisms isolation}

A monitoring operation was carried out from the beginning of March to the end of July during three years: 2011, 2012, and 2013, in fields. Multiple wells were excavated by shovel randomly, and dead larvae were collected (Fig. $1 \mathrm{~A}, \mathrm{~B}, \mathrm{C})$ and taken to the laboratory. Samples were stored at $-20^{\circ} \mathrm{C}$ until processing. For the isolation of potential pathogenic agents, frozen dead larvae were defrosted at room temperature for several minutes, and surface sterilised using $70 \%$ alcohol for $1.5 \mathrm{~min}$. Then, the larvae were subsequently immersed in a $1 \%$ sodium hypochlorite solution for $30 \mathrm{~s}$, rinsed in sterile distilled water three times, and dried on sterile paper towels. Potato Dextrose Agar (PDA, Fulka, Hamburg, Germany) and Nutrient Agar (NA, Merck, Darmstadt, Germany) media were used for the isolation of likely fungal and bacterial pathogens, 


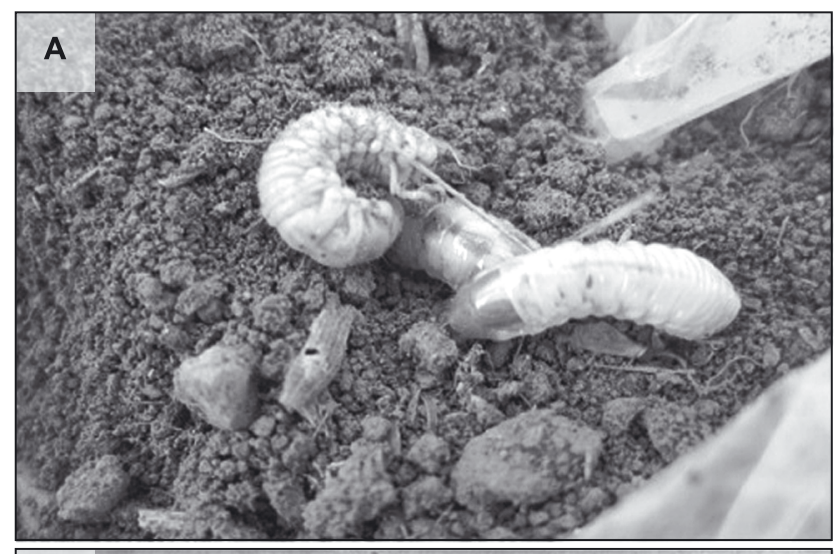

B
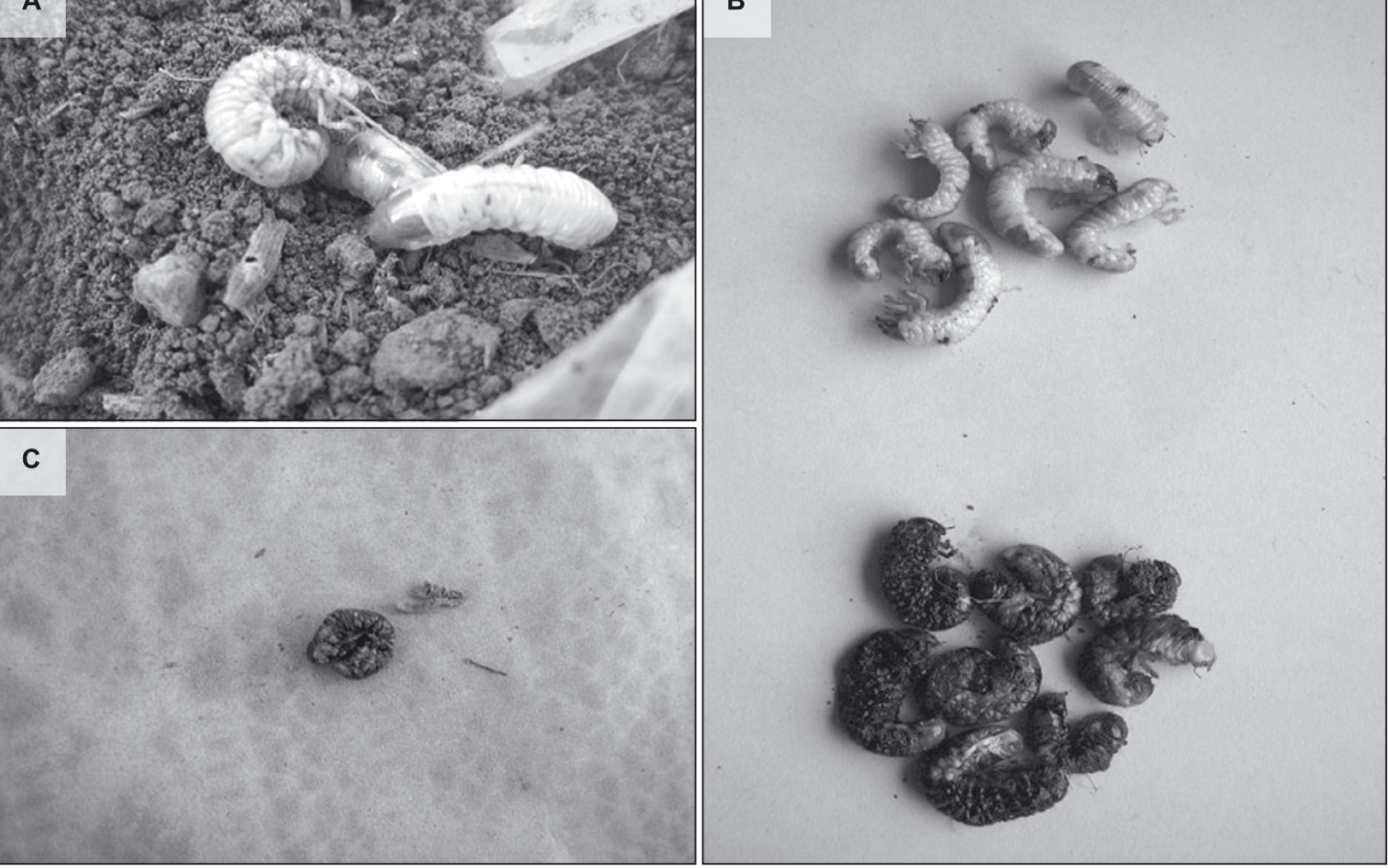

Fig. 1. Chafer beetle mortality caused by Actinomucor elegans var. elegans in wheat fields of Kurdistan province, Iran: A - healthy larvae; B and C - naturally infected larvae of Anisoplia austriaca collected from wheat fields

respectively. For the isolation of the bacteria, disinfected larvae were squashed in sterile distilled water using a sterile scalpel. The resulted suspension was streaked on NA and cultures were incubated at $27^{\circ} \mathrm{C}$ for $48 \mathrm{~h}$. For the isolation of fungal pathogens, disinfected larvae were divided into three parts: the head, chest, and abdomen, and cultured on PDA, then incubated for $48 \mathrm{~h}$ at $30^{\circ} \mathrm{C}$. Pure cultures were deposited in the Culture Collection of Tabriz University (CCTU) for further investigations. Also the fresh culture of CCTU 1815 and CCTU 1816 isolates were deposited at the Iranian Fungal Culture Collection (IRIPP), Tehran, under collection number of IRAN 2361C and IRAN 2362C respectively.

\section{Morphological description}

Cultural characteristics including shape, color, and growth rate of the fungal colony were determined on different culture media including PDA, Czapek solution agar (Merck, Darmstadt, Germany), and Synthetic Nutrient Agar (SNA). Microscopic observations were made using the slide culture technique set up as explained by Arzanlou et al. (2007). For microscopic structures, 30 measurements were made per relevant microscopic feature at $\times 1000$ magnification using an Olympus BX41 light microscope. For the measurements of each structure, 95\% confidence intervals were derived. Photographs of microscopic fungal structures were captured using an Olympus digital DM-21 high definition color camera mounted on the above-mentioned light microscope.

\section{Pathogenicity test (Koch's postulates)}

Koch's postulates were fulfilled using spore suspension and injection methods as described by Cotter et al. (2000) and Sun and Liu (2008). For the spore-suspension method, the recovered fungus was grown on PDA for 4 days at $26^{\circ} \mathrm{C}$. Spore suspension was obtained by flooding cultures with sterile water containing $0.05 \%$ Tween 80 . Mycelium bodies and spores were separated by sterile glass wool. Spore concentration was adjusted at $1 \times 10^{6}$ spores . - $\mathrm{ml}^{-1}$, by hemocytometer. Using forceps, the instar larvae of A. austriaca and G. mellonella (as the model insects) were dipped in spore suspension by forceps for $5 \mathrm{~s}$, and then placed into $9 \mathrm{~cm}$ Petri dishes which contained moistened filter paper (Whatman No. 2). For Galleria larvae, an artificial diet (including flour $600 \mathrm{~g}$, bee wax $60 \mathrm{~g}$, yeast $150 \mathrm{~g}$, honey $300 \mathrm{~g}$, and glycerol $250 \mathrm{ml}$ ) was also added to the Petri dishes. The Petri dishes were sealed by parafilm and kept in darkness at $25^{\circ} \mathrm{C}$. For the controls, instar larvae were dipped in sterile distilled water containing $0.05 \%$ Tween 80 . For the injection test, $20 \mu \mathrm{l}$ of spore suspension was injected into the haemocoel through the hindmost left proleg of G. mellonella larvae only (due to presence of prolegs) by a diabete syringe needle with a diameter of $0.23 \mathrm{~mm}$ (Avapezeshk, Tehran, Iran). For the control, distilled water was injected instead of spore suspension. An additional control, as untreated larvae, was also included. Mortality rate was recorded at $24 \mathrm{~h}$ intervals. Larval death was investigated by the lack of motion of larvae in response to stimulation. Each Petri dish contained five larvae. 


\section{DNA extraction, sequencing and analysis}

Fresh mycelia were harvested from a five-day-old fungal colony grown on PDA. Genomic DNA was extracted using the protocol of Möller et al. (1992). Sequence data from the ITS (Internal Transcribed Spacer) region of ribosomal DNA was used to further confirm the identity of the causal agent. The primers V9G (de Hoog and van den Ende 1998) and LR5 (Vilgalys and Hester 1990) were used to amplify the $3^{\prime}$ end of the 18S rRNA gene, ITS1, 5.8S rDNA, ITS2, and the first approximately $900 \mathrm{bp}$ of the $5^{\prime}$ end of the $28 \mathrm{~S}$ nrRNA gene. For amplification, polymerase chain reaction (PCR) was performed on a Bio-Rad PCR System. The reaction mixture contained $1 \times \mathrm{PCR}$ buffer, $1 \mathrm{mM} \mathrm{MgCl}{ }_{2}$ $60 \mu \mathrm{l}$ of $1 \mathrm{mM}$ dNTPs, $0.2 \mathrm{pM}$ of each primer, $0.5 \mathrm{U}$ of Taq polymerase, $0.5 \mu \mathrm{DMSO}$, and $10-15 \mathrm{ng}$ of fungal genomic DNA. The final reaction volume was adjusted to $15 \mu \mathrm{l}$ by adding sterile distilled water. The thermal cycling condition consisted of an initial denaturation at $95^{\circ} \mathrm{C}$ for $5 \mathrm{~min}$, followed by 40 cycles of $30 \mathrm{~s}$ at $94^{\circ} \mathrm{C}, 30 \mathrm{~s}$ at $52^{\circ} \mathrm{C}$ and $1 \mathrm{~min}$ at $72^{\circ} \mathrm{C}$, followed by a final extension cycle at $72^{\circ} \mathrm{C}$ for $7 \mathrm{~min}$. The amplicon was sequenced using PCR primers and two additional internal primers, ITS4 (White et al. 1990) and LR0R (Rehner and Samuels 1994) in order to obtain good quality sequence over the entire length of the amplicon. A BigDye Terminator Cycle Sequencing Kit v. 3.1 (Applied Biosystems, Foster City, CA) was used, and analyses were done on an ABI Prism 3700 (Applied Biosystems, Foster City, CA). The sequence for the ITS region was subjected to a Megablast search at NCBI's GenBank nucleotide database. Sequences with high similarity were downloaded from GenBank and aligned together with the sequence obtained in this study. Sequence alignment was carried out by using the ClustalW algorithm implemented in MEGA 5 (Tamura et al. 2011). Phylogenetic analysis was performed using the maximum likelihood method. A model of nucleotide substitution was selected using MEGA 5 software as Tamura 3-Parameters (T92) (Tamura et al. 2011). Bootstrap analysis was performed with 10,000 replicates. The phylogenetic tree was rooted to Mucor heimalis (GenBank accession number KF876829.1).

\section{Viability of spores}

The viability of fungal spores was determined by direct enumeration of the germinated spores on medium in Petri dishes containing $16 \mathrm{~g}$ agar, $0.5 \mathrm{~g}$ yeast extract, $0.1 \mathrm{~g}$ Tween 80 , and $20 \mathrm{mg}$ Benlate $50 \mathrm{WP}$ per liter. The prepared medium was poured into $9 \mathrm{~cm}$ Petri dishes in a thin layer $(<5 \mathrm{~mm})$. Spore suspension $(100 \mu \mathrm{l})$ was poured and spread on the medium after incubation for $24 \mathrm{~h}$ at $26^{\circ} \mathrm{C}$ in the darkness. Several plugs of the medium were removed and a drop of lactophenol was added on the surface of each plug. The plugs were then covered with a cover slip. Germination of over $90 \%$ of the spores on the medium surface was considered as a viable culture (Costa et al. 2001). The germination percentage of spores was calculated using the following formula:

$$
\% \text { of germinated spores }=(x / x+y) \times 100 \text {, }
$$

where: $\mathrm{x}$ - germinated spores, $\mathrm{y}$ - ungerminated spores.

\section{Results}

\section{Samples and isolates}

In the first and second monitoring, three and eight infected larvae were collected from wheat fields, respectively (Fig. 1B). White, fast-growing fungal colonies grew from all of the samples on PDA culture medium after 2 days of incubation. No growth was observed on NA culture medium after one month of incubation. All of the isolates showed similar cultural and morphological characteristics. The isolate CCTU 1815 was selected for molecular identification and the pathogenicity test.

\section{Morphological descriptions}

Colonies on PDA and Czapek's agar fast-growing, filled a $9 \mathrm{~cm}$ diameter Petri dish in 4 days at $26^{\circ} \mathrm{C}$. Woolly, floccose colonies forming abundant aerial mycelia, reached the lid of the Petri dish. But no growth was observed at $37^{\circ} \mathrm{C}$. Colony colour on the surface was initially white to grey on both media, with age colour became cream to olive-buff on PDA, and grey on Czapek agar. Colony colour in reverse: white to pale olive-buff and often as cloud-like spots, this appeared to be as a result of rhizoid formation on the medium. Yellow oil droplets were recorded on aerial mycelia. Stolons (5 to $22 \mu \mathrm{m}$ in diameter) and rhizoid were present (Fig. 2G). Branched rhizoids produced abundantly. Sporangiophore originated from stolons (without opposite rhizoid) or rhizoid (Fig. 2I), highly variable in length and width and ending to a sporangium or a set of sporangia. Sporangia formed as primary and secondary in a verticillate manner (Fig. 2B). Primary sporangia were bigger than secondary sporangia. Separation from secondary sporangia was by a cross wall and secondary sporangia separated from sporangiophore and each other by cross walls (Fig. 2B). Sporangia globose to subglobose, buff-color to bright brown, 13 to $75 \mu \mathrm{m}$ in diameter (Fig. 2A), deliquescent or persistent and smooth or spiny, produced on branched and unbranched sporangiophores (Fig. 2C and F). Columellae present in different sizes and shapes, columella of the large spornagia elongate-oval to pyriform, hyaline to faintly coloured, columellae of the secondary sporangia globose to dorsiventrally-flattened. Collarette was often seen (Fig. 2E). Apophysis was not seen. Sporangiospores were smooth to slightly roughened, hyaline, globose to elliptical (3 to $8 \mu \mathrm{m}$ diam) (Fig. 2H). Pleomorphic chlamydospores especially formed in older culture as intercalary single or in short chains, forming an inter-hyphal connection or sporangiophore upon germination (Fig. 2D). Zygospores were absent.

\section{DNA extraction, PCR amplification and sequencing of the ITS region}

After PCR and sequencing of the ITS region, a megablast search at GenBank database showed 100\% similarity with the ITS sequences of other A. elegans (Accession numbers: FJ176396.1, AM745429.1) and one strain of A. elegans var. meitauzae (Accession number: AY492087.1). Next, Phylog- 

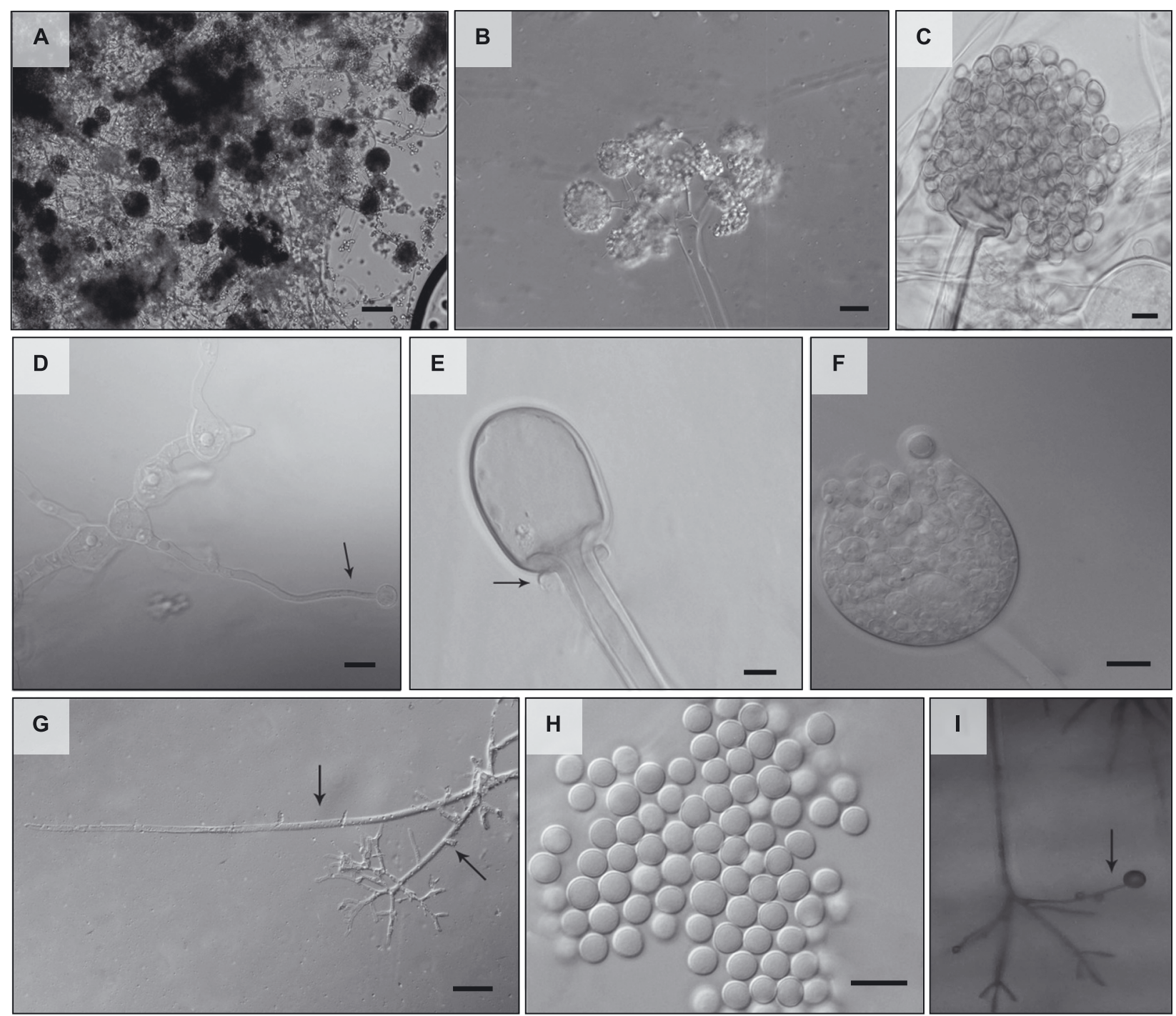

Fig. 2. Actinomucor elegans var. elegans: A - sporangia on PDA; B - primary and secondary sporangia along with sporangiophore; C - sporangium and columella; D - chlamydospores and branched sporangiophores originating from chlamydospores; E - columella and conspicuous collarette underneath (shown by arrow); F - ruptured sporangium along with released sporangiospores and visible columella; G - rhizoid (lower arrow) and stolon (upper arrow); $\mathrm{H}$ - sporangiospores; I - sporangium arising from rhizoid. Bars: A - $50 \mu \mathrm{m} ; \mathrm{B}-20 \mu \mathrm{m} ; \mathrm{C}, \mathrm{D}, \mathrm{E}, \mathrm{F}, \mathrm{H}-10 \mu \mathrm{m} ; \mathrm{G}-100 \mu \mathrm{m}$

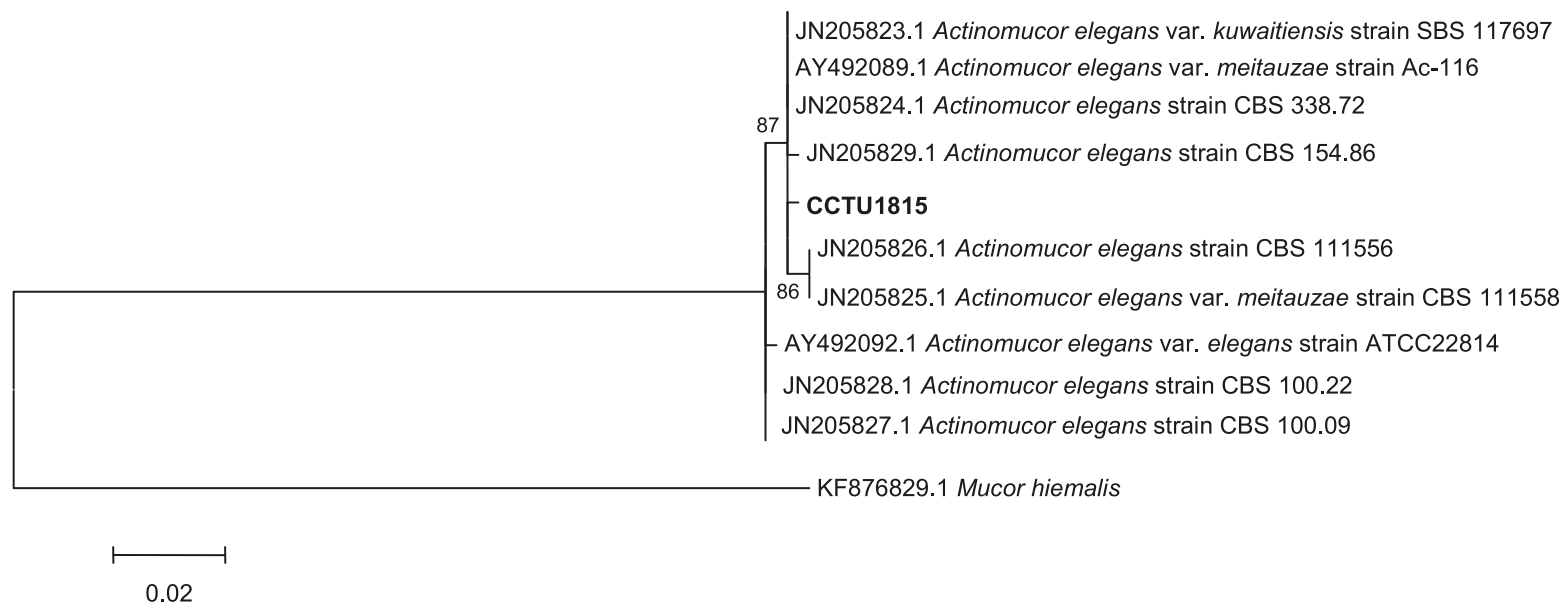

Fig. 3. A maximum likelihood phylogenetic tree based on sequence data from ITS-rDNA region. The scale indicates the number of substitutions per 100 nucleotides. Support for branches was assessed by bootstrap resampling of the data set with 10,000 replications. Tree was rooted by Mucor hiemalis (GenBank accession number KF876829.1). The sequences in boldface text (CCTU 1815) was generated in this study 
Table 1. Pathogenicity assay of Actinomucor elegans var. elegans on instar larvae of Anisoplia austriaca and Galleria mellonella

\begin{tabular}{|c|c|c|c|c|c|c|}
\hline \multirow{2}{*}{ Methods } & \multicolumn{3}{|c|}{ Anisoplia austriaca } & \multicolumn{3}{|c|}{ Galleria mellonella } \\
\hline & dead & alive & MP & dead & alive & MP \\
\hline $\begin{array}{l}\text { Immersion } \\
\text { method }\end{array}$ & 5 & 0 & $100 \%$ & 1 & 4 & $20 \%$ \\
\hline $\begin{array}{l}\text { Injection } \\
\text { method }\end{array}$ & - & - & - & 1 & 4 & $20 \%$ \\
\hline
\end{tabular}

MP - Mortality percentage
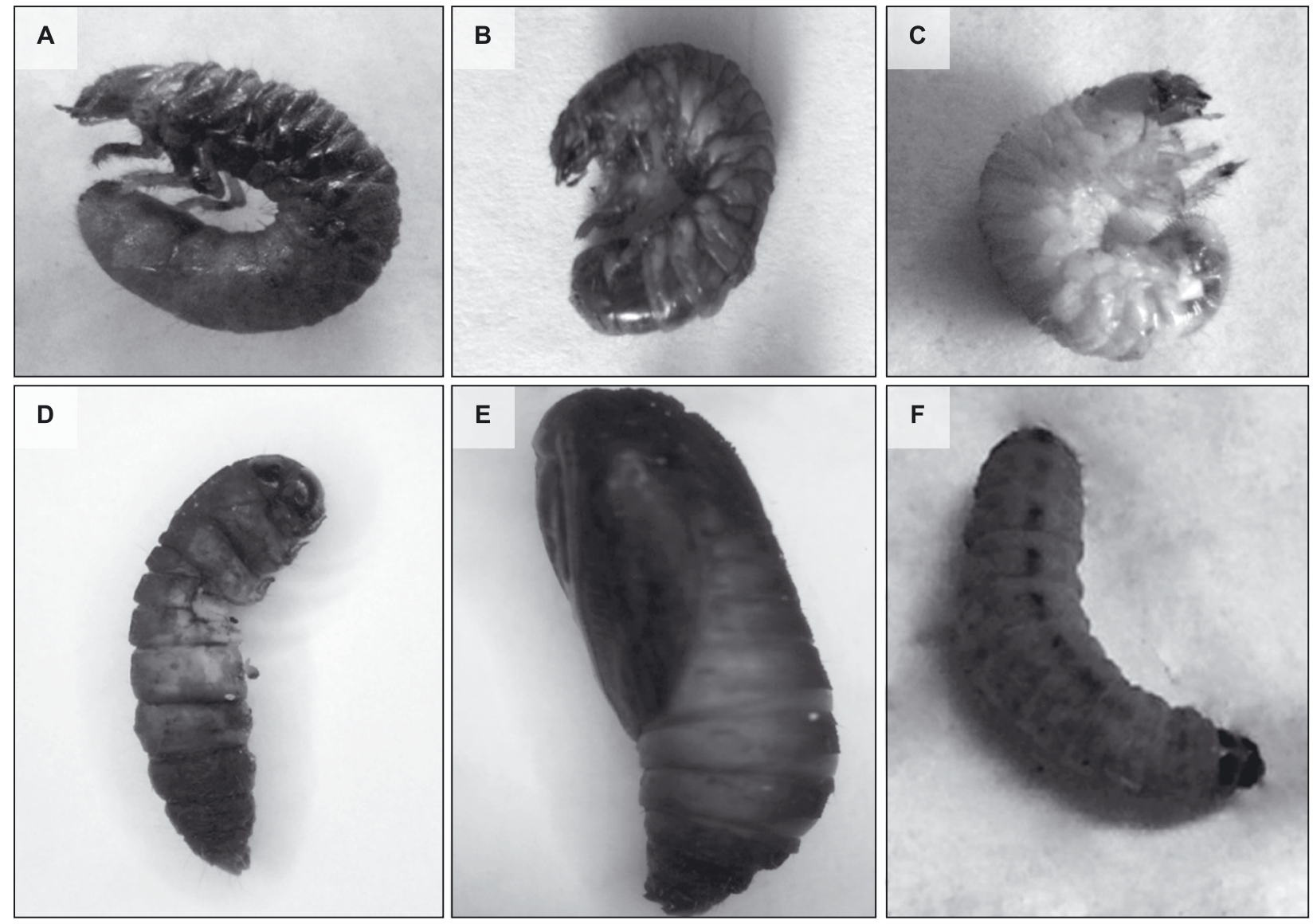

Fig. 4. Pathogenicity assay of Actinomucor elegans var. elegans on instar larvae of Anisoplia austriaca and Galleria mellonella: A and B - dead larvae of A. austriaca using spore suspension method; C - control (intact larva of Anisoplia austriaca); D - dead larva of G. mellonella using injection method; E - dead pupa of G. mellonella using spore suspension; F - control (intact larva of G. mellonella)

Table 2. Results of viability test on Actinomucor elegans var. elegans

\begin{tabular}{|c|c|c|c|c|c|c|c|c|c|c|}
\hline \multirow{2}{*}{ Replications } & \multicolumn{2}{|c|}{ Sample 1} & \multicolumn{2}{|c|}{ Sample 2} & \multicolumn{2}{|c|}{ Sample 3} & \multicolumn{2}{|c|}{ Sample 4} & \multicolumn{2}{|c|}{ Sample 5} \\
\hline & G & UNG & G & UNG & G & UNG & G & UNG & G & UNG \\
\hline Replication 1 & 33 & 3 & 29 & 1 & 21 & 1 & 22 & - & 24 & 2 \\
\hline Replication 2 & 29 & - & 30 & - & 21 & 2 & 25 & - & 32 & 2 \\
\hline Replication 3 & 28 & 2 & 21 & 2 & 23 & - & 28 & - & 24 & 4 \\
\hline MS & \multicolumn{2}{|c|}{$94.99 \%$} & \multicolumn{2}{|c|}{$95.98 \%$} & \multicolumn{2}{|c|}{$95.58 \%$} & \multicolumn{2}{|c|}{$100 \%$} & \multicolumn{2}{|c|}{$90.7 \%$} \\
\hline Total mean & \multicolumn{10}{|c|}{$95.45 \%$} \\
\hline
\end{tabular}

G - Germinated; UNG - Ungerminated; MS - Mean per sample 

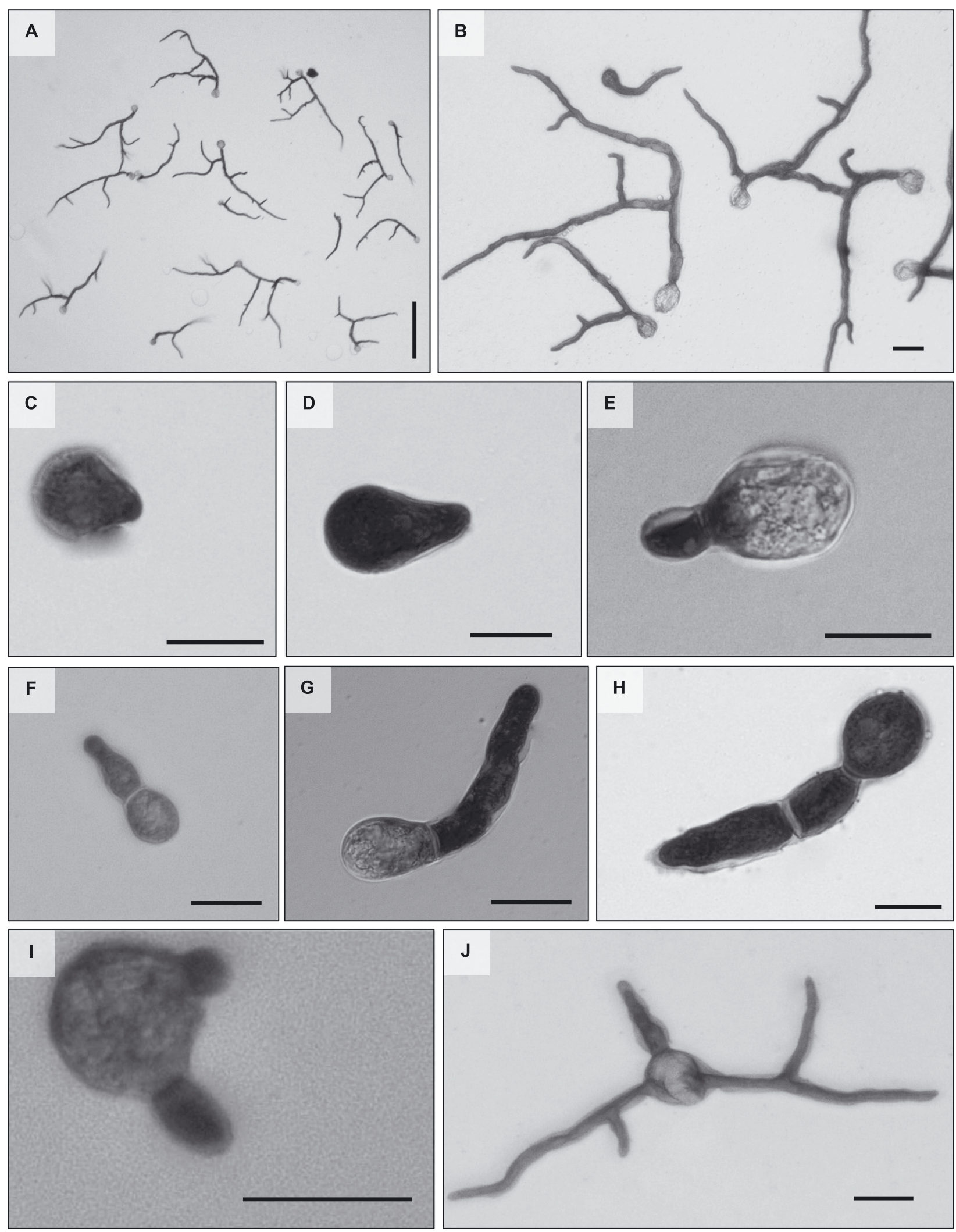

Fig. 5. Viability test of Actinomucor elegans var. elegans isolates: A and B - germinated sporangiospores after $16 \mathrm{~h}$ on yeast extract agar culture media; $\mathrm{C}-\mathrm{J}$ - different patterns of sporangiospore germination among Actinomucor elegans var. elegans isolates. Bars: $\mathrm{A}-100 \mu \mathrm{m} ; \mathrm{B}, \mathrm{F}, \mathrm{I}$ and $\mathrm{J}-20 \mu \mathrm{m} ; \mathrm{C}, \mathrm{D}, \mathrm{E}, \mathrm{G}$ and $\mathrm{H}-10 \mu \mathrm{m}$

eny inferred using the sequence data of ITS region generated in this study, clustered our isolate together with other strains taken from Genbank including A. elegans, A. elegans var. meitauzae, and A. elegans var. kuwaitiensis in high support value (Fig. 3).

\section{Pathogenicity assay}

Treatment of $A$. austriaca larvae with spore suspension resulted in a $100 \%$ mortality rate 4 days after inoculation. However, the mortality rate for G. mellonella larvae was 
lower than the mortality rate for A. austriaca in both suspension and injection methods. Only $20 \%$ of the larvae died after 12 days when the injection method was used and after 16 days when the suspension method was used (Table 1; Fig. 4). To satisfy Koch's postulates, the dead and inoculated samples in all of the treatments were cultured and A. elegans were isolated again from all the samples, except the controls.

\section{Viability test}

Germination of the sporangiospores was examined on yeast extract agar medium (Table 2). Our results revealed a viability mean of $95.45 \%$ (> 90\%) for A. elegans isolates. Sporangiospores were enlarged and swollen nearly two to three fold (Fig. 5A to J) and some of them germinated in two or three directions (Fig. 5I and J).

\section{Discussion}

The results of the pathogenicity assay revealed that $A$. elegans was highly pathogenic on A. austriaca larvae while on G. mellonella larvae, A. elegans showed low pathogenicity. The lower mortality rate on G. mellonella larvae is intriguing, which might indicate that $A$. elegans isolates from $A$. austriaca are host specific. To support this hypothesis, new experiments have to be designed to further evaluate the pathogenicity of A. elegans on other insect species.

The morphological identification of $A$. elegans was further confirmed using sequence data from the ITS-rDNA region. Based on cultural and morphological characteristics, on PDA and Czapek's agar, the fungal isolates were identified as A. elegans. The observations were in full agreement with $A$. elegans features that were previously re-evaluated by Benjamin and Hesseltine (1957). ITS sequence data clearly differentiates Actinomucor from the other morphologically similar genera, such as Mucor, Rhizopus, Rhizomucor, and Absidia, yet, ITS region does not discriminate varieties of $A$. elegans from each other (Walther et al. 2013). In a phylogeny inferred from ITS sequence data, strains with the characteristics of var. meitauzae intermingled with other varieties of nearly all parts of the tree, and only a number of the strains belonging to var. elegans clustered together with the ex-type strain of var. elegans in a well-supported clade (Walther et al. 2013). A combination of morphological and cultural characteristics such as shape, size, ornamentation of the sporangiospores; ability to growth on Czapek's agar and maximum growth temperature (Zheng and Liu 2005; Khan et al. 2008) have been used to distinguish the varieties of A. elegans. On Czapek's agar, the var. meitauzae and var. kuwaitiensis show reduced growth. Contrary to earlier reports (Jong and Yuan 1985), maximum growth temperature is of little help in discriminating the varieties (Zheng and Liu 2005; Khan et al. 2008). The morphological features of our isolates were in agreement with the description of $A$. elegans var. elegans: growing on Czapek's agar, sporangiospores with a size ranging from 3 to $8 \mu \mathrm{m}$, and an inability to grow at $37^{\circ} \mathrm{C}$.

One of the important aspects of biocontrol agents is their spore viability. Spore viability is an innate virulence factor that has a prominent role in determining the pathogenicity of fungal pathogens of insects. For example, reduced conidial viability of Beauveria bassiana has been correlated with the decrease in the mortality rate of the insect host, due to B. bassiana infection (Soetopo 2004). Since our isolates had spore viability higher than $90 \%$, the cultures were considered viable (Milner et al. 1991) (Table 2). Moreover, the other criterion for a spore to be considered viable is the length of the germ tube being twofold of the spore diameter or spore swelling (Herlinda 2010). This characteristic was observed in our experiments (Fig. 5). High spore viability subsequently increases the probability of a viable spore contact by an insect pest when the activity of pest around its environment is after the application (Costa et al. 2001). Swollen or even strongly swollen sporangiospores were recorded in this study. This phenomenon has been observed and reported in some studies for sporangiiferous fungi such as Rhizopus oryzae and Nothadelphia mortierellicola (Waldorf and Diamond 1985; Degawa and Gams 2004).

Mucoralean fungi are ubiquitous, predominantly saprobic soil organisms on decaying organic material. Some species, though, are known to parasite plants, fungi, and animals (Hoffmann et al. 2013). Actinomucor elegans var. elegans is apparently ubiquitous and has been isolated from different hosts and substrates such as rhizosphere of various plants (oat, barley, corn, wheat, alfalfa, and hop), mouse and rabbit dung, nests, feathers and fecal of free-living birds (Phalip et al. 2006; Domsch et al. 2007). Moreover, A. elegans var. elegans along with A. elegans var. kuwaitiensis have been reported as the etiologic agent of mucormycosis in humans in several cases (Davel et al. 2001; Khan et al. 2008; Tully et al. 2009; Mahmud et al. 2012). Another variety, A. elegans var. meitauzae has industrial significance producing various enzymes such as proteolytic and lipolytic enzymes. This variety is frequently used for the production of popular fermented soybean foods including Sufu and Chao, and as a mould starter (Han et al. 2003). Given the results of this study, A. elegans var. elegans can be considered as a potential biocontrol agent against the chafer beetle, owing to its fast growing behavior, abundant production of propagules, and soil inhabitant nature. Actinomucor elegans has previously been isolated from dead bees and termites; however, there is no supplementary data available on the pathogenicity of A. elegans on these hosts (Batra et al. 1973; Zoberi and Grace 1990). To the best of our knowledge, this is first report on the occurrence and pathogenicity of A. elegans var. elegans on the chafer beetle.

\section{Acknowledgements}

The authors wish to thank the Research Deputy of the University of Tabriz for financial support and the Agriculture and Natural Resources Research Center of the Kurdistan province for contribution. We also wish to greatly acknowledge Mr. Narmani and Mr. Chenari for their technical assistance and Ms. Amizadeh and Mr. Asghari for providing Galleria larvae. 


\section{References}

Arzanlou M., Groenewald J.Z., Gams W., Braun U., Shin H.D., Crous P.W. 2007. Phylogenetic and morphotaxonomic revision of Ramichloridium and allied genera. Studies in Mycology 58: 57-93.

Batra L.R., Batra S.W.T., Bohart G.E. 1973. The mycoflora of domesticated and wild bees (Apoidea). Mycopathologia et Mycologia Applicata 49 (1): 13-44.

Benjamin C.R., Hesseltine C.W. 1957. The genus Actinomucor. Mycologia 49: 240-249.

Costa H.S., Robb K.L., Wilen C.A. 2001. Increased persistence of Beauveria bassiana spore viability under high ultravioletblocking greenhouse plastic. HortScience 36 (6): 1082-1084.

Cotter G., Doyle S., Kavanagh K. 2000. Development of an insect model for the in vivo pathogenicity testing of yeasts. FEMS Immunology \& Medical Microbiology 27 (2): 163-169.

Davel G., Featherston P., Fernández A., Abrantes R., Cristina C., Rodero L., Sztern C., Perrotta D. 2001. Maxillary sinusitis caused by Actinomucor elegans. Journal of Clinical Microbiology 39 (2): 740-742.

de Hoog G.S., Gerrits van den Ende A.H.G. 1998. Molecular diagnostics of clinical strains of filamentous Basidiomycetes. Mycoses 41 (5-6): 183-189.

Degawa Y., Gams W. 2004. A new species of Mortierella, and an associated sporangiiferous mycoparasite in a new genus, Nothadelphia. Studies in Mycology 50 (2): 567-572.

Domsch K.H., Gams W., Anderson T.H. 2007. Compendium of Soil Fungi. IHW-Verlag, Eching, Netherlands, 43 pp.

Han B.Z., Ma Y., Rombouts F.M., Nout M.J.R. 2003. Effects of temperature and relative humidity on growth and enzyme production by Actinomucor elegans and Rhizopus oligosporus during sufu pehtze preparation. Food Chemistry 81 (1): 27-34.

Herlinda S. 2010. Spore density and viability of entomopathogenic fungal isolates from Indonesia, and their virulence against Aphis gossypii Glover (Homoptera: Aphididae). Trop Life Sciences Results 21 (1): 11-19.

Hoffmann K., Pawłowska J., Walther G., Wrzosek M., de Hoog G.S., Benny G.L., Kirk P.M., Voigt K. 2013. The family structure of the Mucorales: a synoptic revision based on comprehensive multigene-genealogies. Persoonia 30: 57-76.

Jameson M., Mico E., Galante E. 2007. Evolution and phylogeny of the scarab subtribe Anisopliina (Coleoptera: Scarabaeidae: Rutelinae: Anomalini). Systematic Entomology 32 (3): 429-449.

Jong S.C., Yuan G.F. 1985. Actinomucor taiwanensis sp. nov., for manufacture of fermented soybean food. Mycotaxon 23: 261-264.

Khan Z.U., Ahmad S., Mokaddas E., Chandy R., Cano J., Guarro J. 2008. Actinomucor elegans var. kuwaitiensis isolated from the wound of a diabetic patient. Antonie Van Leeuwenhoek 94 (3): 343-352.

Klein M.G., Kaya H.K. 1995. Bacillus and Serratia a species for scarab control. Memórias do Instituto Oswaldo Cruz 90 (1): 87-95.
Mahmud A., Lee R., Munfus-McCray D., Kwiatkowski N., Subramanian A., Neofytos D., Carroll K., Zhang S.X. 2012. Actinomucor elegans as an emerging cause of Mucormycosis. Journal of Clinical Microbiology 50 (3): 1092-1095.

Milner R.J., Huppatz R J., Swaris S.C. 1991. A new method for assessment of germination of Metarhizium conidia. Journal of Invertebrate Pathology 57 (1): 121-123.

Möller E.M., Bahnweg G., Geiger H.H. 1992. A simple and efficient protocol for isolation of high molecular weight DNA from filamentous fungi, fruit bodies, and infected plant tissues. Nucleic Acids Results 20 (22): 6115-6116.

Phalip V., Hatsch D., Laugel B., Jeltsch J.M. 2006. An overview of fungal community diversity in diseased hop plantations. FEMS Microbiology Ecology 56 (2): 321-329.

Rehner S.A., Samuels G.J. 1994. Taxonomy and phylogeny of Gliocladium analysed from nuclear large subunit ribosomal DNA sequences. Mycological Research 98 (6): 625-634.

Soetopo D. 2004. Efficacy of selected Beauveria bassiana (Bals.) Vuill. isolates in combination with a resistant cotton variety (PSB-Ct 9) against the cotton bollworm, Helicoverpa armigera (Hübner) (Lepidoptera: Noctuidae). Ph.D. thesis, University of the Philippines Los Banos, Philippines.

Sun B.D., Liu X.Z. 2008. Occurrence and diversity of insect-associated fungi in natural soils in China. Applied Soil Ecology 39 (1): 100-108.

Tamura K., Peterson D., Peterson N., Stecher G., Nei M., Kumar S. 2011. MEGA5: Molecular Evolutionary Genetics Analysis using maximum likelihood, evolutionary distance, and maximum parsimony methods. Molecular Biology and Evolution 28 (10): 2731-2739.

Tully C.C., Romanelli A.M., Sutton D.A., Wickes B.L., Hospenthal D.R. 2009. Fatal Actinomucor elegans var. kuwaitiensis infection following combat trauma. Journal of Clinical Microbiology 47 (10): 3394-3399.

Vilgalys R., Hester M. 1990. Rapid genetic identification and mapping of enzymatically amplified ribosomal DNA from several Cryptococcus species. Journal of Bacteriology 172 (8): 4238-4246.

Waldorf A.R., Diamond R.D. 1985. Neutrophil chemotactic responses induced by fresh and swollen Rhizopus oryzae spores and Aspergillus fumigatus conidia. Infection and Immunity 48 (2): 458-463.

Walther G., Pawłowska J., Alastruey-Izquierdo A., Wrzosek M., Rodriguez-Tudela J.L., Dolatabadi S., Chakrabarti A., de Hoog G.S. 2013. DNA barcoding in Mucorales: an inventory of biodiversity. Persoonia 30: 11-47.

White T.J., Bruns T.D., Lee S.B., Taylor J.W. 1990. Amplification and sequencing of fungal ribosomal RNA genes for phylogenetics. p. 315-322. In: "PCR-Protocols and Applications, a Laboratory Manual" (N. Innis, D. Gelfand, J. Sninsky, T.C. White, eds.). Academic Press, New York, USA.

Zheng R.Y., Liu X.Y. 2005. Actinomucor elegans var. meitauzae, the correct name for A. taiwanensis and Mucor meitauzae (Mucorales, Zygomycota). Nova Hedwigia 80 (3-4): 419-431.

Zoberi M.H., Grace J.K. 1990. Fungal associated with the subterranean termite Reticulitermes flavipes in Ontario. Mycologia 82 (3): 289-294. 